\title{
Serum neurofilament light chain level as a predictor of cognitive stage transition
}

Eun-Hye Lee ${ }^{1 \dagger}$, Hyuk Sung Kwon ${ }^{1 \dagger}$, Seong-Ho Koh ${ }^{1 *} \mathbb{D}$, Seong Hye Choi ${ }^{2 *}$, Jeong-Hwa Jin ${ }^{1}$, Jee Hyang Jeong ${ }^{3}$, Jae-Won Jang ${ }^{4}$, Kyung Won Park ${ }^{5}$, Eun-Joo Kim ${ }^{6}$, Hee Jin Kim ${ }^{7}$, Jin Yong Hong ${ }^{8}$, Soo Jin Yoon ${ }^{9}$, Bora Yoon ${ }^{10}$, Ju-Hee Kang ${ }^{11}$, Jong-Min Lee ${ }^{12}$, Hyun-Hee Park ${ }^{1}$ and Jungsoon $\mathrm{Ha}^{1,13}$

\begin{abstract}
Background: Neurofilament light chain (NFL) level has been suggested as a blood-based biomarker for neurodegeneration in dementia. However, the association between baseline NFL levels and cognitive stage transition or cortical thickness is unclear. This study aimed to investigate whether baseline NFL levels are associated with cognitive stage transition or cortical thickness in mild cognitive impairment (MCl) and cognitively unimpaired (CU) participants.

Methods: This study analyzed data on participants from the independent validation cohort of the Korea Brain Aging Study for the Early Diagnosis and Prediction of Alzheimer's disease (KBASE-V) study. Among the participants of KBASE$V$ study, $53 \mathrm{MCl}$ and $146 \mathrm{CU}$ participants who were followed up for $\geq 2$ years and had data on the serum NFL levels were eligible for inclusion in this study. Participants were classified into three groups according to baseline serum NFL levels of low, middle, or high.

Results: The Kaplan-Meier analysis showed association between the serum NFL tertiles and risk of cognitive stage transition in $\mathrm{MCl}(P=0.002)$ and $\mathrm{CU}(P=0.028)$ participants, analyzed separately. The same is true upon analysis of $\mathrm{MCl}$ and $\mathrm{CU}$ participants together $(P<0.001)$. In MCl participants, the highest serum NFL tertile and amyloid-beta positivity were independent predictors for cognitive stage transition after adjusting for covariates. For CU participants, only amyloid-beta positivity was identified to be an independent predictor.

Conclusion: The study shows that higher serum NFL tertile levels correlate with increased risk of cognitive stage transition in both $\mathrm{MCl}$ and CU participants. Serum NFL levels were negatively correlated with the mean cortical thickness of the whole-brain and specific brain regions.
\end{abstract}

Keywords: Neurofilament light chain, Alzheimer's disease, Cognitive stage, Amyloid pathology, Cortical thickness

\section{Background}

Alzheimer's disease (AD) is the most frequent cause of dementia. Globally, the number of individuals with dementia has been increasing [1]. The cascade of AD is

\footnotetext{
*Correspondence: ksh213@hanyang.ac.kr; seonghye@inha.ac.kr †Eun-Hye Lee and Hyuk Sung Kwon contributed equally to this work. 1 Department of Neurology, Hanyang University Guri Hospital, Hanyang University College of Medicine, 153 Gyeongchun-ro, Guri 11923, Republic of Korea

2 Department of Neurology, Inha University School of Medicine, 27 Inhang-ro, Jung-gu, Incheon, Republic of Korea

Full list of author information is available at the end of the article
}

believed to begin with extracellular accumulation of amyloid-beta $(A \beta)$, which leads to intracellular formation of neurofibrillary tangles, synaptic dysfunction, neuronal loss, and cognitive decline [2,3]. It is important to define biomarkers that best predict the progression of $\mathrm{AD}$ [2]. To identify individuals who are likely to progress to the clinical stage of $\mathrm{MCI}$ and $\mathrm{AD}$ at an early stage, many advances pertaining to $\mathrm{AD}$ biomarkers have been made. For example, brain atrophy and $A \beta$ positivity can precede symptoms by years to decades [2]. However, these biomarkers are assessed by magnetic resonance imaging original author(s) and the source, provide a link to the Creative Commons licence, and indicate if changes were made. The images or other third party material in this article are included in the article's Creative Commons licence, unless indicated otherwise in a credit line to the material. If material is not included in the article's Creative Commons licence and your intended use is not permitted by statutory regulation or exceeds the permitted use, you will need to obtain permission directly from the copyright holder. To view a copy of this licence, visit http://creativecommons.org/licenses/by/4.0/. The Creative Commons Public Domain Dedication waiver (http://creativeco mmons.org/publicdomain/zero/1.0/) applies to the data made available in this article, unless otherwise stated in a credit line to the data. 
(MRI), positron emission tomography (PET) amyloid imaging, or cerebrospinal fluid (CSF) studies, which are expensive and/or invasive. In addition, it is unclear whether individuals with brain atrophy and/or $A \beta$ positivity will develop dementia during their lifetime. Individual differences among clinicians in interpreting these biomarkers (degree of brain atrophy and $A \beta$ positivity) are also limitations. Recently, neurofilament light chain (NFL) level has been proposed as a blood biomarker that can overcome these limitations [4].

NFL levels in the CSF are related to neuronal death and axonal degeneration [5]. Plasma NFL levels are higher in individuals with mild cognitive impairment (MCI) or Alzheimer's disease dementia (ADD) than in cognitively unimpaired (CU) individuals [4]. Moreover, there is a significant correlation between NFL levels in the CSF and the blood [6]. Higher plasma NFL levels have also been associated with poor cognition, brain atrophy, and brain hypometabolism [4]. In familial AD, serum NFL levels were predictive of the rate of cortical thinning and cognitive decline [6]. A meta-analysis revealed that NFL levels in the blood and the CSF could not differentiate AD from disease mimics including vascular dementia, Lewy body dementia, Parkinson's disease dementia, idiopathic normal pressure hydrocephalus, and posterior cortical atrophy [7]. Taken together, increased NFL levels may play an important role in stratifying individuals with early-stage dementia and those who are likely to show cognitive stage transition. However, the role of NFL as a blood biomarker of dementia to identify individuals who are likely to show cognitive stage transition is unclear. In addition, the association between serum NFL levels and cortical thickness of specific brain regions is unclear.

Therefore, we investigated whether serum NFL levels are associated with cognitive stage transition in $\mathrm{CU}$ or MCI individuals over a 3-year period. The clinical characteristics of the participants, according to the serum NFL tertile and the association between the serum NFL levels and cortical thickness of each specific brain region, were analyzed.

\section{Methods}

\section{Participants}

This study analyzed data on participants from the independent validation cohort of the Korean Brain Aging Study for the Early Diagnosis and Prediction of AD (KBASE-V) [8]. The KBASE-V contains a nationwide cohort, including $167 \mathrm{CU}, 72 \mathrm{MCI}$, and $56 \mathrm{ADD}$ participants from nine hospitals across South Korea from April 2015 to August 2016. The participants were between 55 and 90 years of age. Among these participants, the eligible patients for the current study were those with (1) $\mathrm{CU}$ or MCI, (2) more than 2 years of follow-up, and (3) serum NFL level data. In total, $146 \mathrm{CU}$ and $53 \mathrm{MCI}$ participants were included in this study.

All CU participants had normal ( $\geq 1.5$ standard deviations [SDs] below the age-, sex-, and education-adjusted normative means) performance on four memory tests of the Korean version of the Consortium to Establish a Registry for Alzheimer's Disease (CERAD; word list immediate recall, word list delayed recall, word list recognition, and constructional praxis recall) and had a global Clinical Dementia Rating (CDR) scale score of 0 [9-11]. MCI participants met the core clinical criteria for $\mathrm{MCI}$ due to $\mathrm{AD}$ established by the National Institute on Aging-Alzheimer's Association (NIA-AA) workgroups [12] and the following criteria modified from the criteria proposed by Petersen et al. [13]: (1) CDR scale score of 0.5, (2) memory complaints by patients, caregivers, or clinicians; (3) a performance score $<1.5$ SDs below the age-, education-, and sex-adjusted normative means for one or more of the four memory tests included in the CERAD, (4) the ability to perform independent activities of daily living (ADL) [14], and (5) absence of dementia. All participants were aged between 55 and 90 years and had a reliable informant who could provide the requested information to the investigators. The exclusion criteria were as follows: (1) presence of major psychiatric illness, (2) significant neurological or medical conditions or comorbidities that could affect cognitive function, (3) contraindications for MRI (e.g., pacemaker and claustrophobia), (4) illiteracy, (5) severe visual or hearing difficulty or serious communication or behavioral problems that could hinder clinical examination or brain imaging; (6) receiving an investigational drug; and (7) pregnancy or breastfeeding [8].

\section{Clinical assessment}

All participants underwent yearly physical and neurological examinations including thorough diagnostic procedures that assessed participants' cognition, abnormal behaviors, ADL, demographic characteristics, family history, current medications, vascular risk factors (the presence of hypertension, diabetes, dyslipidemia and smoking status), and other comorbidities using the MiniMental State Examination (MMSE) [9], Geriatric Depression Scale (Gdps) [15], Blessed Dementia Scale-ADL [16], CDR scale, and CERAD $[8,17]$. Brain MRI and laboratory tests, including blood biochemistry assessment, lipid panel, complete blood count test, folate test, vitamin B12 test, venereal disease research laboratory test, thyroid function test, and apolipoprotein E (APOE) genotyping, were performed at baseline. Participants' weight and height were measured while they were wearing light clothing. Participants' body mass index (BMI) was calculated using their weight $(\mathrm{kg})$ divided by the square of their height $\left(\mathrm{m}^{2}\right)$. 


\section{Brain MRI}

All participants underwent brain MRI. A 3.0-T MRI scanner was used to capture three-dimensional (3D) T1- and T2-weighted SPACE sagittal images with $0.8-\mathrm{mm}$ thickness. AD Neuroimaging Initiative phase 2 MRI protocols were used for brain MRI $[8,18]$. The 3D T1-weighted MRI parameters were as follows: repetition time (TR) = $2300 \mathrm{~ms}$, echo time $(\mathrm{TE})=2.14 \mathrm{~ms}$, inversion time (TI) $=900 \mathrm{~ms}$, flip angle $(\mathrm{FA})=9^{\circ}$, and voxel resolution $=0.8$ $\times 0.8 \times 0.8 \mathrm{~mm}^{3}$ in the Skyra and Trio Tim scanners (Siemens, Washington, DC, USA); TR $=7.32 \mathrm{~ms}$, TE $=3.02$ $\mathrm{ms}$, $\mathrm{TI}=400 \mathrm{~ms}, \mathrm{FA}=11^{\circ}$, and voxel resolution $=0.8 \times$ $0.8 \times 0.8 \mathrm{~mm}^{3}$ in the General Electric Discovery MR750 scanner (GE Healthcare, Milwaukee, WI, USA); and TR $=$ shortest $(6.8 \mathrm{~ms}), \mathrm{TE}=$ shortest $(3.1 \mathrm{~ms}), \mathrm{FA}=9^{\circ}$, and voxel resolution $=0.8 \times 0.8 \times 0.8 \mathrm{~mm}^{3}$ in the Achieva scanner (Philips Healthcare, Andover, MA, USA).

The measured MRI data were analyzed using CIVET pipeline version 2.1 (https://mcin.ca/technology/civet/) [19]. The intensity difference from inhomogeneity in the magnetic field was calibrated using the N3 intensity nonuniformity correction algorithm, and the corrected T1-weighted images were aligned to the Montreal Neurological Institute 152 standard space $[20,21]$. The BET algorithm was adjusted to exclude non-brain tissue from the data [22]. The inner and outer surfaces of the cortex were estimated using a deformable spherical mesh and constrained Laplacian-based automated segmentation with proximities algorithm, respectively [23]. The cortical thickness values in the native space were obtained using the Euclidean distance between the linked vertices of the inner and outer surfaces [24]. The corrected T1-weighted images were segmented into the left and right sides of the hippocampus using FMRIB's integrated registration and segmentation tool [25]. The volumes of the hippocampus were normalized for the total intracranial volume.

\section{Positive amyloid pathological change (A $\beta$ biomarkers)}

Amyloid pathological change was considered positive when individuals had an abnormal A $\beta$ biomarker based on cortical amyloid PET ligand binding and/or low CSF A 342 levels [26]. In total, 159 (79.9\%) participants underwent amyloid PET at baseline. Sixty participants underwent ${ }^{11} \mathrm{C}$-PiB PET and 99 participants underwent ${ }^{18}$ F-flutemetamol PET. The CSF was collected from 100 (50.3\%) participants. In total, 184 (92.5\%) participants underwent testing for A $\beta$ biomarkers, of whom 49 (24.6\%) were positive.

The PET methods for each tracer and CSF analysis have been previously described [8, 17]. The standard uptake value ratio (SUVR) was obtained using the pons as a reference region on ${ }^{18} \mathrm{~F}$-flutemetamol PET and the cerebellar gray matter as the reference region on ${ }^{11} \mathrm{C}-\mathrm{PiB}$ PET. The Centiloid replication analysis was performed according to previous reports $[27,28]$. Based on a previous study, elevated $A \beta$ PET was defined as a cut-point of 10 Centiloid units $[28,29]$. The levels of $A \beta 42$, $t$-tau, and p-tau in the CSF were measured using the multiplex xMAP Luminex platform with INNO-BIA AlzBio3 kits (Fujirebio Europe, Ghent, Belgium). The method has been described in detail in a previous paper [8]. Based on a previous study, participants who underwent CSF studies were deemed to have AD pathology when the CSF A 342 level was $\leq 433.68 \mathrm{pg} / \mathrm{mL}$ [8].

\section{NFL}

CSF NFL levels were measured using NF-light ELISA RUO kit according to the instructions provided by UmanDiagnostics. Serum NFL levels were estimated using SIMOA NF-light Advantage kit produced from Quanterix. The serum and CSF samples were prepared and provided by the nine medical centers participating in the validation cohort of the KBASE-V, including Inha University Hospital. Briefly, to measure the NFL levels, CSF samples were reacted with a biotinylated detection antibody for $1 \mathrm{~h}$. The detected antigen was captured by the streptavidin-HRP complex, and then, the TMB substrate was added and incubated for 20 min under protection from light. The NFL signal was measured at $450 \mathrm{~nm}$.

\section{Outcome}

The main outcome was the cognitive stage transition (from CU to MCI or dementia and from MCI to dementia) during the 3-year study period. The diagnosis of MCI was based on the core clinical criteria for MCI established by the NIA-AA workgroups [12] and the criteria modified from the criteria proposed by Petersen et al. [13], as mentioned earlier $[8,17]$. The diagnosis of dementia was based on the DSM-IV-TR criteria for dementia [30], and the diagnosis of probable ADD was based on the NIAAA core clinical criteria [31].

\section{Statistical analysis}

Patients were divided into three groups based on tertiles of serum NFL levels at baseline. Pearson's chi-square test, one-way analysis of variance, or the Kruskal-Wallis test was used to compare the variables among each group. When statistically significant overall differences were detected, Pearson's chi-square test with Bonferroni correction or Tukey's post hoc comparisons was performed to examine differences among subgroups. The time to the outcome was assessed using the Kaplan-Meier method and between-group outcome was compared by the logrank tests. We assessed the univariate and multivariate effects of covariates using Cox proportional hazards 
models to estimate the relative hazards of cognitive stage transition in MCI and CU participants assessed separately and then together. Adjusted variables were age, sex, and factors selected from the results of the univariate analysis with $P<0.05$. In addition, variables regarded as potential confounders (serum NFL [tertile], age, sex, education level, baseline MMSE score, initial cognitive stage, hippocampal volume, $\mathrm{A} \beta$ positivity, APOE $\varepsilon 4$ genotype, cortical thickness, BMI, and co-morbid hypertension, DM, or dyslipidemia) were adjusted as covariates in the analysis of total (MCI plus CU) participants. The participants were divided into two groups according to the presence of cognitive stage transition. Pearson's chisquare test, Student's $t$ test, and the Mann-Whitney $U$ test were used to evaluate differences between the two groups. Nonparametric correlation between the cortical thickness of specific brain regions and serum NFL levels was calculated using Spearman's test as cortical thickness and serum NFL levels were not normally distributed (Kolmogorov-Smirnov test, $P<0.01$ ). A two-tailed $p$ value of $<0.05$ was considered statistically significant. All statistical analyses were performed using SPSS for Windows version 21.0 (SPSS Inc., Chicago, IL, USA).

\section{Results}

This study included 199 (146 CU and $53 \mathrm{MCI}$ ) participants aged 55-90 years (mean age \pm SD: $69.3 \pm$ 8.3 years). The follow-up period ranged from 23 to 49 months (mean \pm SD: $33.4 \pm 7.6$ months). A total of 36 (18.1\%) participants showed cognitive stage transition over the study period. Among the $146 \mathrm{CU}$ participants, 14 (9.6\%) participants progressed to $\mathrm{MCI}$ and two (1.4\%) participants progressed to dementia. Among the 53 MCI participants, 19 (35.8\%) participants progressed to

Table 1 Baseline characteristics of the MCI participants according to the initial serum NFL levels

\begin{tabular}{|c|c|c|c|c|}
\hline & $\begin{array}{l}\text { Lowest tertile } \\
(n=18)\end{array}$ & $\begin{array}{l}\text { Middle tertile } \\
(n=18)\end{array}$ & $\begin{array}{l}\text { Highest tertile } \\
(n=17)\end{array}$ & $P$-value* \\
\hline Mean serum NFL, pg/ml & $12.9 \pm 4.0^{c}$ & $21.2 \pm 3.4^{b}$ & $39.7 \pm 15.5^{a}$ & $<0.001^{* *}$ \\
\hline Range of serum NFL & $5.6-18.3$ & $18.4-23.8$ & $24.0-84.5$ & \\
\hline \multicolumn{5}{|l|}{ Demographics } \\
\hline Age, years & $67.8 \pm 7.0^{b}$ & $72.8 \pm 7.6^{b}$ & $79.4 \pm 6.3^{\mathrm{a}}$ & $<0.001^{* *}$ \\
\hline Sex, female & $9(50.0)$ & $8(44.4)$ & $6(35.3)$ & $0.676^{*}$ \\
\hline Lives alone & $4(22.2)$ & $1(5.6)$ & $3(17.6)$ & $0.354^{*}$ \\
\hline $\mathrm{BMI}, \mathrm{kg} / \mathrm{m}^{2}$ & $24.0 \pm 2.4$ & $24.2 \pm 2.8$ & $24.4 \pm 2.4$ & $0.920^{* *}$ \\
\hline Education, years & $9.4 \pm 3.6$ & $9.3 \pm 4.4$ & $9.2 \pm 4.3$ & $0.993^{* *}$ \\
\hline MMSE score, median (IQR) & $25.0(22.0-27.25)$ & $24.0(20.0-26.0)$ & $21.0(19.5-23.5)$ & $0.041+$ \\
\hline CDR score, median (IQR) & $1.0(1.0-1.0)$ & $1.0(1.0-1.0)$ & $1.0(1.0-1.0)$ & $0.591+$ \\
\hline CDR-SOB score, median (IQR) & $1.0(1.0-1.25)$ & $1.0(1.0-2.0)$ & $2.0(1.0-2.0)$ & $0.129+$ \\
\hline Gdps score, median (IQR) & $8.5(4.75-13.25)$ & $10.0(3.0-18.75)$ & $9.0(4.0-13.0)$ & $0.727 \dagger$ \\
\hline \multicolumn{5}{|l|}{ Medical history } \\
\hline Hypertension & $7(38.9)$ & $9(50.0)$ & $9(52.9)$ & $0.677^{*}$ \\
\hline Diabetes mellitus & $4(22.2)$ & $2(11.1)$ & $5(29.4)$ & $0.618 \neq$ \\
\hline Dyslipidemia & $7(38.9)$ & $7(38.9)$ & $1(5.9)$ & $0.045^{*}$ \\
\hline Coronary artery disease & $2(11.1)$ & $1(5.6)$ & $0(0.0)$ & $0.159 \neq$ \\
\hline Cerebrovascular disease & $0(0.0)$ & $1(5.6)$ & $1(5.9)$ & $0.362 \ddagger$ \\
\hline Smoking & $1(5.6)$ & $0(0.0)$ & $1(5.9)$ & $0.974 \neq$ \\
\hline Pill intake of more than three & $10(55.6)$ & $10(55.6)$ & $9(52.9)$ & $0.984^{*}$ \\
\hline Hippocampal volume, $\mathrm{cm}^{3}$ & $5.1 \pm 0.7^{a}$ & $4.3 \pm 1.4^{b}$ & $4.4 \pm 0.5^{a b}$ & $0.033^{* *}$ \\
\hline Cortical thickness, mm & $3.01 \pm 0.15$ & $3.10 \pm 0.17$ & $3.07 \pm 0.11$ & $0.212^{* *}$ \\
\hline$A \beta$ positivity & $7 / 17(41.2)$ & $11 / 17(64.7)$ & $8 / 15(53.3)$ & $0.389 *$ \\
\hline APOE ع4 carrier & $3(16.7)$ & $6(33.3)$ & $3(17.6)$ & $0.928 \neq$ \\
\hline Family history of dementia & $5(274.8)$ & $6(33.3)$ & $5(29.4)$ & $0.933^{*}$ \\
\hline Cognitive stage transition & $1(5.6)^{b}$ & $7(38.9)^{a b}$ & $11(64.7)^{\mathrm{a}}$ & $0.001^{*}$ \\
\hline
\end{tabular}

Data are presented as mean \pm standard deviation or number (\%), unless otherwise indicated

NFL neurofilament light chain, MMSE Mini-Mental State Examination, IQR interquartile range, CDR-SOB Clinical Dementia Rating Scale Sum of Boxes, Gdps Geriatric Depression Scale, $A \beta$ amyloid-beta

Based on *Pearson's chi-square test, ${ }^{* *}$ analysis of variance, the tKruskal-Wallis test, or flinear by linear association

$a>b>c$. Pearson's chi-square test with Bonferroni correction or Tukey's post hoc comparisons was performed to assess for significant differences among the subgroups 
dementia. Of the 184 (92.5\%) participants who underwent testing for $\mathrm{A} \beta$ biomarkers, 50 (25.1\%) were $\mathrm{A} \beta$ positive. APOE genotyping was performed in all cases; 43 (21.6\%) participants were APOE $\varepsilon 4$ carriers.

The clinical characteristics of the $\mathrm{CU}$ and MCI participants, according to serum NFL levels, are described in Tables 1 and 2. In the MCI group, individuals with higher serum NFL tertiles were more likely to be older and showed greater tendency to have dyslipidemia, lower hippocampal volume, lower MMSE scores, and higher risk for cognitive stage transition (Table 1). In the analysis of both the CU group alone and MCI plus CU together (total participants), serum NFL tertile was found to be associated with age, education, lower MMSE score, pill intake of more than three, cortical thickness, and cognitive stage transition (Table 2 and Supplementary Table 1). There were no significant differences in sex, live alone,
BMI, CDR score, Gdps score, coronary artery disease, cerebrovascular disease, cortical thickness, $A \beta$ positivity, and APOE $\varepsilon 4$ carrier among three groups. The KaplanMeier analysis showed graded associations between the serum NFL tertiles and risk of cognitive stage transition in $\mathrm{MCI}(P=0.002$, Fig. $1 \mathrm{~A}), \mathrm{CU}(P=0.028$, Fig. $1 \mathrm{~B})$, and total $(P<0.001$, Fig. $1 C)$ participants.

In the univariate Cox proportional hazards regression analysis, serum NFL tertile, age, baseline MMSE score, hippocampal volume, and $A \beta$ positivity were found to be associated with cognitive stage transition in $\mathrm{MCI}, \mathrm{CU}$, and total (MCI plus CU) participants (Table 3).

The multivariate Cox proportional hazards regression analysis adjusted for covariates which include serum NFL tertiles, age, sex, baseline MMSE score, hippocampal volume, and $A \beta$ positivity, the highest serum NFL tertile (hazard ratio [HR] 13.640, 95\% confidence interval [CI]

Table 2 Baseline characteristics of the CU participants according to the initial serum NFL levels

\begin{tabular}{|c|c|c|c|c|}
\hline & $\begin{array}{l}\text { Lowest tertile } \\
(n=49)\end{array}$ & $\begin{array}{l}\text { Middle tertile } \\
(n=49)\end{array}$ & $\begin{array}{l}\text { Highest tertile } \\
(n=48)\end{array}$ & $P$-value* \\
\hline Mean serum NFL, pg/ml & $11.9 \pm 2.6^{c}$ & $19.1 \pm 2.3^{b}$ & $37.2 \pm 13.8^{\mathrm{a}}$ & $<0.001^{* *}$ \\
\hline Range of serum NFL, pg/ml & $6.2-15.0$ & $15.1-22.6$ & $22.7-77.8$ & \\
\hline \multicolumn{5}{|l|}{ Demographics } \\
\hline Age, years & $63.2 \pm 6.4^{b}$ & $69.2 \pm 7.3^{\mathrm{a}}$ & $71.3 \pm 7.3^{\mathrm{a}}$ & $<0.001^{* *}$ \\
\hline Sex, female & $36(73.5)$ & $30(61.2)$ & $24(50.0)$ & $0.059^{*}$ \\
\hline Lives alone & $7(14.3)$ & $7(14.3)$ & $7(14.6)$ & $0.999^{*}$ \\
\hline $\mathrm{BMI}, \mathrm{kg} / \mathrm{m}^{2}$ & $24.2 \pm 2.6$ & $25.0 \pm 3.5$ & $24.5 \pm 2.8$ & $0.469^{* *}$ \\
\hline Education, years & $12.9 \pm 3.8^{\mathrm{a}}$ & $10.7 \pm 5.3^{a b}$ & $8.8 \pm 5.3^{b}$ & $<0.001^{* *}$ \\
\hline MMSE score, median (IQR) & $28.0(27.0-29.0)^{\mathrm{a}}$ & $28.0(25.5-29.0)^{a}$ & $21.0(19.5-23.5)^{b}$ & $<0.001 \dagger$ \\
\hline CDR score, median (IQR) & $0.0(0.0-0.0)$ & $0.0(0.0-0.0)$ & $0.0(0.0-0.0)$ & $0.372 \dagger$ \\
\hline CDR-SOB score, median (IQR) & $0.0(0.0-0.0)$ & $0.0(0.0-0.0)$ & $0.0(0.0-0.0)$ & $0.045+$ \\
\hline Gdps score, median (IQR) & $7.0(3.5-12.5)$ & $6.0(2.5-10.5)$ & $8.0(4.25-14.0)$ & $0.241+$ \\
\hline \multicolumn{5}{|l|}{ Medical history } \\
\hline Hypertension & $16(32.7)$ & $24(49.0)$ & $26(56.5)$ & $0.057^{*}$ \\
\hline Diabetes mellitus & $4(8.2)$ & $6(12.2)$ & $12(25.0)$ & $0.054^{*}$ \\
\hline Dyslipidemia & $18 / 48(37.5)$ & $22(44.9)$ & $21 / 46(45.7)$ & $0.673^{*}$ \\
\hline Coronary artery disease & $1(2.0)$ & $3(6.1)$ & $4(8.3)$ & $0.174 \neq$ \\
\hline Cerebrovascular disease & $1(2.0)$ & $1(2.0)$ & $5(10.4)$ & $0.055 \neq$ \\
\hline Smoking & $2(4.1)$ & $0(0.0)$ & $3(6.3)$ & $0.565 \neq$ \\
\hline Pill intake of more than three & $40(81.6)^{a}$ & $27(55.1)^{b}$ & $24(50.0)^{b}$ & $0.003^{*}$ \\
\hline Hippocampal volume, $\mathrm{cm}^{3}$ & $5.2 \pm 0.9$ & $5.0 \pm 0.7$ & $5.0 \pm 0.8$ & $0.365^{* *}$ \\
\hline Cortical thickness, mm & $3.13 \pm 0.12^{\mathrm{a}}$ & $3.10 \pm 0.14^{\mathrm{ab}}$ & $3.04 \pm 0.16^{b}$ & $0.007^{* *}$ \\
\hline$A \beta$ positivity & $6 / 45(13.3)$ & $11 / 45(24.4)$ & $7 / 45$ (15.6) & $0.345^{*}$ \\
\hline APOE ع4 carrier & $11(22.4)$ & $11(22.4)$ & $9(18.8)$ & $0.877^{*}$ \\
\hline Family history of dementia & $15(30.6)$ & $10(20.4)$ & $12(25.0)$ & $0.508^{*}$ \\
\hline Cognitive stage transition & $1(2.0)^{b}$ & $6(12.2)^{a b}$ & $9(18.8)^{\mathrm{a}}$ & $0.029^{*}$ \\
\hline
\end{tabular}

Data are presented as mean \pm standard deviation or number (\%), unless otherwise indicated

NFL neurofilament light chain, MMSE Mini-Mental State Examination, IQR interquartile range, CDR-SOB Clinical Dementia Rating Scale Sum of Boxes, Gdps Geriatric Depression Scale, $A \beta$ amyloid-beta

Based on *Pearson's chi-square test, ${ }^{* *}$ analysis of variance, the tKruskal-Wallis test, or flinear by linear association

$a>b>c$. Pearson's chi-square test with Bonferroni correction or Tukey's post hoc comparisons was performed to assess for significant differences among the subgroups 
A Mild cognitive impairment patients $(n=53)$

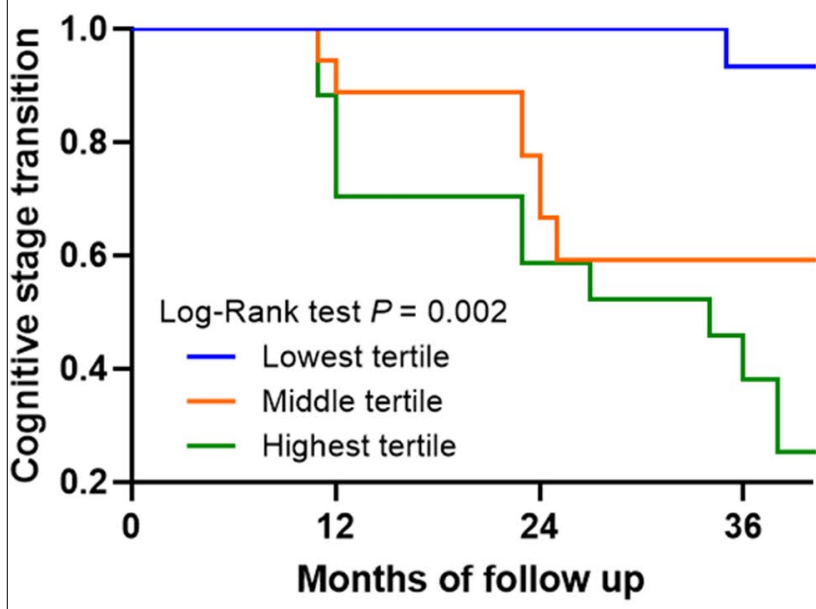

B Cognitive Unimpaired participants $(n=146)$

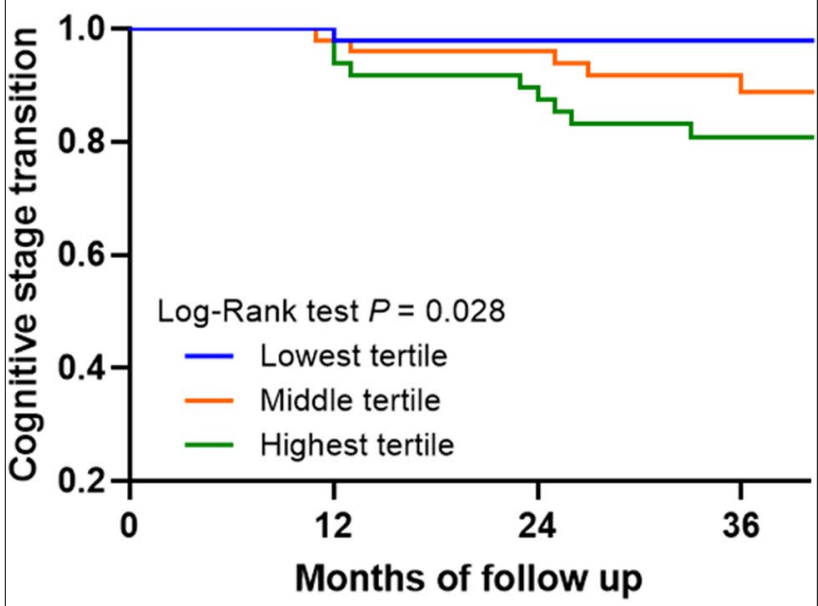

C Total participants $(n=199)$

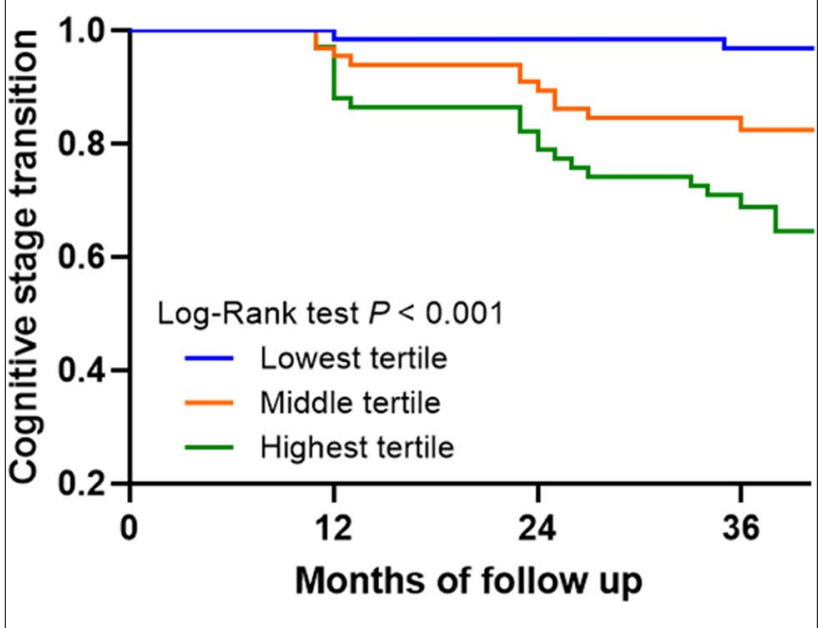

Fig. 1 Cognitive stage transition. Kaplan-Meier curves for cognitive stage transition according to the serum NFL tertile in participants with mild cognitive impairment (A), cognitively unimpaired (B), and total participants (C)

1.346-138.270, $P=0.027$ ) and $\mathrm{A} \beta$ positivity (HR 7.647, 95\% CI 2.041-28.654, $P=0.003$ ) were independent predictors of cognitive stage transition in MCI participants (Table 4). For CU participants, only A $\beta$ positivity (HR 10.244, 95\% CI 2.996-35.026, $P<0.001$ ) was identified to be an independent predictor. In total (MCI plus $\mathrm{CU}$ ) participants, the highest serum NFL tertile (HR 6.346, 95\% CI 1.345-29.937, $P=0.020$ ), baseline MMSE score (HR 0.866, 95\% CI 0.785-0.955, $P=0.004$ ), and A $\beta$ positivity (HR8.848, 95\% CI 3.614-21.662, $P<0.001$ ) were independent predictor. After additional adjustment for potential confounders (education, initial cognitive stage, APOE $\varepsilon 4$ carrier, cortical thickness, hypertension, diabetes mellitus, dyslipidemia, and BMI), NFL tertile, baseline MMSE score, and $A \beta$ positivity remained as significant predictors (Supplementary Table 2).

Table 5 shows the demographic characteristics of the participants and their risk factors according to cognitive stage transition (i.e., CU to MCI or dementia and MCI to dementia) during the study period. Participants with cognitive stage transition tend to be older and were more likely to have diabetes, lower cognitive function, lower hippocampal volume, more $A \beta$ positivity, and a higher serum NFL tertile than those without cognitive stage transition. Among participants with cognitive stage transition, 20 of 32 (62.5\%) participants had $A \beta$ positivity. Older age, lower cognitive function, lower hippocampal volume, more $A \beta$ positivity, and a higher serum NFL tertile were noted in participants with cognitive stage transition in both the MCI and CU groups (Supplementary Tables 3 and 4).

Serum NFL levels were negatively correlated with the average whole-brain cortical thickness and cortical thickness of the parietal lobe, temporal lobe, and occipital lobe (Fig. 2 and Supplementary Figure 1). The cortical thickness of specific brain regions, including the hippocampus, globus pallidus, accumbens, putamen, and thalamus, were also significantly negatively correlated with serum NFL levels (Fig. 2 and Supplementary Table 5). No specific brain regions were positively correlated with serum NFL levels. Correlation between serum NFL levels and cortical thickness in MCI and CU participants, separately, are demonstrated in Supplementary Figures 2 and 3. In MCI participants, serum NFL levels were negatively correlated with the thickness of the calcarine fissure and surrounding cortex, lingual gyrus, accumbens, 
Table 3 Univariate Cox regression analysis of cognitive stage transition

\begin{tabular}{|c|c|c|c|}
\hline & $\begin{array}{l}\mathrm{MCl} \\
(n=53)\end{array}$ & $\begin{array}{l}\mathrm{CU} \\
(n=146)\end{array}$ & $\begin{array}{l}\text { Total } \\
(n=199)\end{array}$ \\
\hline \multicolumn{4}{|l|}{ Serum NFL } \\
\hline Lowest tertile & $1.00[$ ref $]$ & 1.00 [ref] & 1.00 [ref] \\
\hline Middle tertile & $9.265(1.136-75.587)^{*}$ & $4.968(0.580-42.529)$ & $6.171(1.379-27.609)^{*}$ \\
\hline Highest tertile & $19.569(2.133-128.682)^{* *}$ & $9.660(1.224-76.272)^{*}$ & $11.385(2.664-48.648)^{* *}$ \\
\hline Age & $1.118(1.043-1.199)^{* *}$ & $1.081(1.013-1.154)^{*}$ & $1.119(1.070-1.171)^{* *}$ \\
\hline Sex & $0.911(0.364-2.280)$ & $1.927(0.698-5.316)$ & $1.599(0.822-3.111)$ \\
\hline Lives alone & $1.612(0.529-4.908)$ & $1.530(0.432-5.425)$ & $1.522(0.662-3.498)$ \\
\hline BMI & $0.863(0.711-1.047)$ & $1.022(0.866-1.206)$ & $0.942(0.831-1.068)$ \\
\hline Education & $1.016(0.903-1.143)$ & $0.927(0.842-1.022)$ & $0.951(0.888-1.018)$ \\
\hline Baseline MMSE score & $0.818(0.730-0.916)^{* *}$ & $0.805(0.702-0.923)^{* *}$ & $0.788(0.729-0.852)^{* *}$ \\
\hline Initial GDS score & 1.019 (0.949-1.094) & $1.068(0.997-1.144)$ & $1.054(1.004-1.106)^{*}$ \\
\hline Hypertension & $1.219(0.494-3.008)$ & $1.007(0.365-2.778)$ & $1.202(0.619-2.333)$ \\
\hline Diabetes mellitus & $1.031(0.341-3.112)$ & $4.099(1.457-11.533)$ & $2.073(0.987-4.354)$ \\
\hline Dyslipidemia & $0.246(0.057-1.071)$ & $0.857(0.305-2.408)$ & $0.429(0.195-0.944)^{*}$ \\
\hline Pill intake of more than three & $0.968(0.392-2.386)$ & $0.662(0.240-1.828)$ & $0.718(0.366-1.408)$ \\
\hline Hippocampal volume, per $1 \mathrm{~cm}^{3}$ & $0.628(0.447-0.882)^{* *}$ & $0.666(0.448-0.989)^{*}$ & $0.600(0.477-0.756)^{* *}$ \\
\hline Cortical thickness, per $1 \mathrm{~mm}$ & $4.090(0.226-73.875)$ & $0.059(0.002-1.574)$ & $0.325(0.032-3.288)$ \\
\hline$A \beta$ positivity & $5.436(1.553-19.030)^{* *}$ & $10.724(3.584-32.091)^{* *}$ & $10.306(4.594-23.124)^{* *}$ \\
\hline APOE $\varepsilon 4$ carrier & $2.546(0.981-6.661)$ & $1.932(0.660-5.653)$ & $2.137(1.057-4.3192)^{*}$ \\
\hline Family history of dementia & $1.343(0.526-3.430)$ & $2.085(0.742-5.858)$ & $1.826(0.914-3.648)$ \\
\hline
\end{tabular}

Data are presented as $\mathrm{HR}(95 \% \mathrm{Cl})$

$M C I$ mild cognitive impairment, $C U$ cognitive unimpaired, NFL neurofilament light chain, $B M /$ body mass index, MMSE Mini-Mental State Examination, GDS geriatric depression scale, $H R$ hazard ratio, $A \beta$ amyloid-beta

${ }^{*} P<0.05,{ }^{* *} P<0.01$

Table 4 Multivariate Cox regression analysis of cognitive stage transition

\begin{tabular}{llll}
\hline & MCI & CU & Total \\
\hline Serum NFL & & & \\
$\quad$ Lowest tertile & $1.00[$ ref $]$ & $1.00[$ ref $]$ & $1.00[$ ref $]$ \\
$\quad$ Middle tertile & $5.771(0.593-56.175)$ & $1.954(0.196-19.535)$ & $3.541(0.737-17.019)$ \\
$\quad$ Highest tertile & $13.640(1.346-138.270)^{*}$ & $3.982(0.443-35.809)$ & $6.346(1.345-29.937)^{*}$ \\
Age & $1.030(0.940-1.804)$ & $0.918(0.921-1.095)$ & $1.007(0.953-1.064)$ \\
Sex & $0.443(0.109-1.804)$ & $1.888(0.636-5.598)$ & $1.496(0.694-3.226)$ \\
Baseline MMSE score & $0.925(0.799-1.070)$ & $0.849(0.700-1.030)$ & $0.866(0.785-0.955)^{*}$ \\
Hippocampal volume, per $1 \mathrm{~cm}^{3}$ & $0.703(0.392-1.260)$ & $0.977(0.499-1.911)$ & $0.899(0.609-1.327)$ \\
Aß positivity & $7.647(2.041-28.654)^{*}$ & $10.244(2.996-35.026)^{* *}$ & $8.848(3.614-21.662)^{* *}$ \\
\hline
\end{tabular}

Data are presented as hazard ratio $(95 \% \mathrm{Cl})$

$M C l$ mild cognitive impairment, $C U$ cognitive unimpaired, $N F L$ neurofilament light chain, MMSE Mini-Mental State Examination, $A \beta$ amyloid-beta

${ }^{*} P<0.05,{ }^{* *} P<0.01$

Adjusted for serum NFL (tertile), age, sex, baseline MMSE score, hippocampal volume, amyloid pathology

hippocampus, and putamen. In contrast, serum NFL levels were negatively correlated with the average whole-brain cortical thickness and cortical thickness of the parietal lobe, temporal lobe, occipital lobe, and most of the other specific brain regions in $\mathrm{CU}$ participants.

\section{Discussion}

The present study demonstrated that a higher serum NFL tertile was associated with cognitive stage transition in $\mathrm{CU}$ and MCI participants. After adjusting for covariates, it was found that high serum NFL tertile levels and $A \beta$ positivity were independent predictors of cognitive stage 
Table 5 Clinical characteristics of participants based on cognitive stage transition over the study period

\begin{tabular}{|c|c|c|c|}
\hline & $\begin{array}{l}\text { Non-converter } \\
(n=164)\end{array}$ & $\begin{array}{l}\text { Converter } \\
(n=35)\end{array}$ & $P$-value \\
\hline \multicolumn{4}{|l|}{ Demographics } \\
\hline Age, years & $67.9 \pm 7.8$ & $75.9 \pm 7.2$ & $<0.001^{* *}$ \\
\hline Female & $97(59.1)$ & $16(45.7)$ & $0.145^{*}$ \\
\hline Lives alone & $22(13.4)$ & $7(20.0)$ & $0.316^{*}$ \\
\hline $\mathrm{BMI}, \mathrm{kg} / \mathrm{m}^{2}$ & $24.5 \pm 2.9$ & $24.0 \pm 2.5$ & $0.363^{* *}$ \\
\hline Education, years & $10.6 \pm 4.8$ & $9.4 \pm 5.4$ & $0.192^{* *}$ \\
\hline MMSE score, median (IQR) & $27.0(24.0-29.0)$ & $23.0(20.0-26.0)$ & $<0.001 \neq$ \\
\hline CDR score, median (IQR) & $0.0(0.0-0.0)$ & $0.0(0.0-0.5)$ & $0.001 \neq$ \\
\hline $\begin{array}{l}\text { CDR-SOB score, median } \\
\text { (IQR) }\end{array}$ & $0.0(0.0-0.0)$ & $0.5(0.0-1.0)$ & $<0.001 \neq$ \\
\hline Gdps score, median (IQR) & $7.0(4.0-11.75)$ & $11.0(3.0-14.0)$ & $0.090 \neq$ \\
\hline Initial cognitive stage & & & $<0.001^{*}$ \\
\hline Cognitive unimpaired & $130(79.3)$ & $49(45.7)$ & \\
\hline $\begin{array}{l}\text { Mild cognitive impair- } \\
\text { ment }\end{array}$ & $34(20.7)$ & $19(54.3)$ & \\
\hline \multicolumn{4}{|l|}{ Medical history } \\
\hline Hypertension & $73 / 162(45.1)$ & $18(51.4)$ & $0.493^{*}$ \\
\hline Diabetes mellitus & $23(14.0)$ & $10(28.6)$ & $0.036^{*}$ \\
\hline Dyslipidemia & $68 / 161(42.2)$ & $8(22.9)$ & $0.033^{*}$ \\
\hline Coronary artery disease & $9(5.5)$ & $2(5.7)$ & $1.000+$ \\
\hline Cerebrovascular disease & $6(3.7)$ & $3(8.6)$ & $0.197 \dagger$ \\
\hline Smoking & $6(3.7)$ & $1(2.9)$ & $1.000+$ \\
\hline $\begin{array}{l}\text { Pill intake of more than } \\
\text { three }\end{array}$ & $101(61.6)$ & $19(54.3)$ & $0.451^{*}$ \\
\hline Hippocampal volume, $\mathrm{cm}^{3}$ & $5.1 \pm 0.8$ & $4.4 \pm 1.0$ & $<0.001^{* *}$ \\
\hline Cortical thickness, mm & $3.08 \pm 0.15$ & $3.05 \pm 0.14$ & $0.289^{*}$ \\
\hline $\begin{array}{l}\text { Positive amyloid pathol- } \\
\text { ogył }\end{array}$ & 27/152 (17.8) & 23/32 (71.9) & $<0.001^{*}$ \\
\hline APOE \&4 carrier & $31(18.9)$ & $12(34.3)$ & $0.045^{*}$ \\
\hline Family history of dementia & $43(26.2)$ & $13(37.1)$ & $0.393^{*}$ \\
\hline $\begin{array}{l}\text { Initial serum NFL level, } \\
\text { tertile }\end{array}$ & & & $<0.001^{*}$ \\
\hline Lowest tertile & $64(39.0)$ & $2(5.7)$ & \\
\hline Middle tertile & $54(32.9)$ & $12(34.3)$ & \\
\hline Highest tertile & $46(28.0)$ & $21(60.0)$ & \\
\hline
\end{tabular}

IQR interquartile range, NFL neurofilament light chain

Data are presented as mean \pm standard deviation or number (\%). unless otherwise indicated

Based on *Pearson's chi-square test, ${ }^{*}$ Student's t-test, the †Fisher's exact test, or ¥Mann-Whitney $U$ test

transition in MCI participants alone or MCI plus CU participants together. Serum NFL levels were negatively correlated with the cortical thickness of the whole-brain and specific brain regions, including the hippocampus, thalamus, and basal ganglia.

Progression of dementia is associated with age, baseline cognitive function [32], education level [33], hippocampal volume [34], A $\beta$ positivity [2], and vascular risk factors [35]. However, to our knowledge, this is the first study to report that baseline serum NFL levels can help predict cognitive stage transition in total (CU and $\mathrm{MCI}$ ) participants after adjusting for covariates, including the aforementioned factors. According to a recent study on presymptomatic familial AD, serum NFL levels were predictive of cognitive decline [6]. However, it is important to apply these findings to sporadic AD. In our study, $71.9 \%$ participants had no family history of dementia. Longitudinal plasma NFL levels have been suggested as a noninvasive biomarker for monitoring neurodegeneration in patients with $\mathrm{AD}$ and the effects of drugs in clinical trials [36]. Our study demonstrates that serum NFL levels may play an important role, alone or in combination with other biomarkers, to differentiate converters from non-converters.

There have been several clinical trials on $\mathrm{AD}$ treatments, but no treatment has been shown to modify the progression of neurodegeneration. One of the reasons for the failure these clinical trials is that they were not performed early enough to modify the disease process [37]. In addition, cognitive decline may not occur in some individuals with $A \beta$ positivity [2]. Currently, biomarkers, including brain atrophy and $A \beta$ positivity, are assessed using MRI, PET, or CSF studies to determine individuals who are likely to progress to ADD. However, assessment of these biomarkers is expensive and/or invasive. The use of serum NFL levels as a biomarker for neurodegenerative diseases can help overcome some of the limitations of the current biomarkers. Establishing additional biomarkers will help to identify a strong link between the combination of biomarkers and the progression of clinical symptoms in the early stage of AD.

Neurofilaments are a structural component of perikaryal, dendrites, and large myelinated axons [38, 39]. They are considered potential surrogate biomarkers of diverse neurodegenerative diseases [40]. Neurofilaments are heteropolymers requiring three subunits-light, medium, and heavy [39]. Increased NFL levels in the CSF of patients with AD are associated with neuronal death and axonal degeneration [5]. In this study, serum NFL levels were negatively correlated with the cortical thickness of the parietal, temporal, and occipital lobes, which is in line with a the finding of a previous study that showed an association between higher baseline plasma NFL levels and a decline in cortical thickness [41]. However, to our knowledge, an association between serum NFL levels and the cortical thickness of specific brain regions has not been reported previously. Regarding the volume of specific brain regions, its relationship with NFL levels differed among previous reports. In a study of genetic frontotemporal dementia, NFL levels in the CSF were negatively correlated with the volume of the 
Correlation Coefficient with Serum NFL

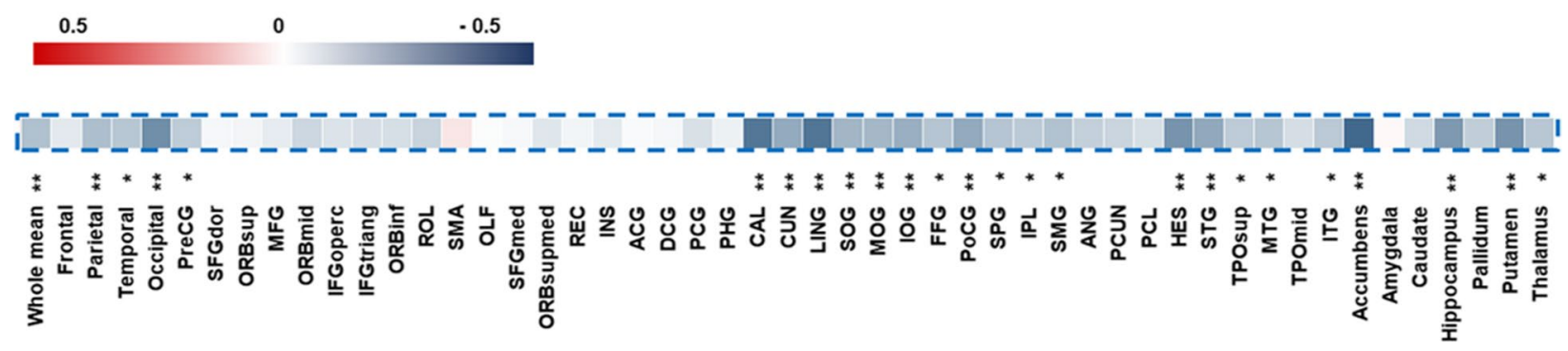

Fig. 2 The correlation between the cortical thickness and serum NFL levels. The correlation between the cortical thickness of specific brain regions and serum NFL levels is represented using different colors. The positive and negative correlation coefficients are shown in red and blue, respectively. Statistical significance was analyzed using Spearman's bivariate correlation $\left({ }^{*} p\right.$ value $<0.05$, ${ }^{* *} p$ value $\left.<0.01\right)$. PreCG, precentral gyrus; SFGdor, superior frontal gyrus (dorsal); ORBsup superior orbital gyrus; MFG, middle frontal gyrus; ORBmid, middle orbital gyrus; IFGoperc, inferior frontal gyrus pars opercularis; IFGtriang, inferior frontal gyrus pars triangularis; ORBinf, inferior orbital gyrus; ROL, rolandic operculum; SMA, supplementary motor area; OLF, olfactory cortex, SFGmed, superior frontal gyrus (medial); ORBsupmed, superior frontal gyrus (medial orbital); REC, gyrus rectus; INS, insula; ACG, anterior cingulate gyrus; DCG, dorsal cingulate gyrus; PCG, posterior cingulate gyrus; PHG, parahippocampal gyrus; CAL, calcarine fissure and surrounding cortex; CUN, cuneus; LING, lingual gyrus; SOG, superior occipital gyrus; MOG, middle occipital gyrus; IOG, inferior occipital gyrus; FFG, fusiform gyrus; PoCG, postcentral gyrus; SPG, superior parietal gyrus; IPL, inferior parietal lobule; SMG, supra marginal gyrus; ANG, angular gyrus; PCUN, precuneus; PCL, paracentral lobule; HES, Heschl's gyrus; STG, superior temporal gyrus; TPOsup, superior temporal pole; MTG, middle temporal gyrus; TPOmid, middle temporal pole; ITG, inferior temporal gyrus

frontal, parietal, temporal, insular, and cingulate cortices, but they were positively correlated with the volume of the occipital cortex [42]. Another study demonstrated that baseline and longitudinal plasma NFL levels were associated with the composite volume of the hippocampus and entorhinal and temporal cortices [36]. The reason for the different associations between serum NFL levels and specific brain regions is unclear. NFL levels may reflect the degree of neurodegeneration in any brain region [40], rather than having a deep relationship with specific brain regions.

Furthermore, in our study, highest serum NFL tertile in individuals with negative amyloid pathology were associated with cognitive stage transition $(P=0.012$, Supplementary Table 4). None of the individuals with both negative amyloid pathology and the lowest serum NFL tertile showed cognitive stage transition. These results indicate that NFL level may be a biomarker that nonspecifically reflects neurodegeneration and is not diseasespecific to AD. A previous study has also suggested that NFL level might be a general biomarker for axonal degeneration rather than a tool to differentiate $\mathrm{AD}$ from other types of dementia [4]. However, measuring NFL levels may help screen out individuals who are likely to show cognitive stage transition.

This study has several limitations. First, participants of a single ethnicity were included. Second, the median follow-up period was only 3 years. This short follow-up period might be insufficient to show the difference in the relationship of cognitive stage transition and serum NFL tertile in CU participants. Thus, the relationship between serum NFL levels and cognitive stage transition was found to be more prominent in the MCI group than in the $\mathrm{CU}$ group. Including both normal and subjective cognitive decline participants in the $\mathrm{CU}$ group and more participants with a family history of dementia in the MCI group than in the CU group $(32.1 \%$ vs. $26.7 \%$, respectively) might be other reasons for this difference. However, all participants in this study were followed up for more than 2 years, which could help determine the specific target in clinical trials. Third, some non-amnestic MCI patients might have been classified into the $\mathrm{CU}$ group due to the inclusion criteria of scoring above age-, sex-, and education-adjusted memory scales. However, these criteria are in line with Alzheimer's disease neuroimaging initiative 3 protocol [43]. Also, all CU participants in current study showed normal cognitive function in all domains except two participants who showed performance scores that were $<1.5$ SDs below the normative means for visuospatial function. Fourth, we allowed three 3D T1-weighted MRI parameters in this cohort. Although the difference from inhomogeneity in the magnetic field was calibrated using the N3 intensity nonuniformity correction algorithm, there might be remaining differences depending on scanner types that we failed to consider. Fifth, serum NFL levels were only measured at baseline. However, it would be useful to predict cognitive stage transition using serum NFL levels measured at single time point. Finally, NFL levels in the CSF were measured in only half $(n=100)$ of the participants. However, the correlation coefficient between the CSF and serum NFL levels was $0.358(p<0.001$, Supplementary Figure 4). 


\section{Conclusions}

In conclusion, high serum NFL levels in MCI and CU participants indicated that they had a high risk of cognitive stage transition. Furthermore, serum NFL levels were negatively correlated with the cortical thickness of the whole-brain and specific brain regions.

\section{Abbreviations}

AD: Alzheimer's disease; ADD: Alzheimer's disease dementia; BMI: Body mass index; CDR: Clinical dementia rating; CU: Cognitively unimpaired; DM: Diabetes mellitus; GE: General electric; MCl: Mild cognitive impairment; MMSE: MiniMental State Examination; NCEP: National cholesterol education program; OR: Odds ratio; SD: Standard deviation; SUVR: Standard uptake value ratio.

\section{Supplementary Information}

The online version contains supplementary material available at https://doi. org/10.1186/s13195-021-00953-x.

Additional file 1: Supplementary Table 1. Baseline characteristics of the total participants according to the initial serum NFL levels. Supplementary Table 2. Multivariate Cox regression analysis of the factors for cognitive stage transition in total participants. Supplementary Table 3. Clinical characteristics of participants based on cognitive stage transition over the study period in mild cognitive impairment participants $(n=53)$. Supplementary Table 4. Clinical characteristics of participants based on cognitive stage transition over the study period in cognitively unimpaired participants $(n=146)$. Supplementary Table 5 . Correlation between cortical thickness and neurofilament light chain levels in the serum. The cortical thickness of specific brain regions, including the hippocampus, globus pallidus, accumbens, putamen, and thalamus, were significantly negatively correlated with serum NFL levels. Supplementary Table 6. Clinical characteristics of participants with negative amyloid pathology according to the initial serum NFL levels $(n=135)$. Higher serum NFL levels in individuals with negative amyloid pathology were associated with cognitive stage transition $(P=0.003)$.

Additional file 2: Supplementary Figure 1. The correlation between the cortical thickness of each specific brain region and serum NFL. The correlation between the cortical thickness of each specific brain region and serum NFL is represented in different colors. The positive and negative correlation coefficients are shown in red and blue, respectively. The cortical thickness between each brain region showed a positive correlation. NFL levels were negatively correlated with the cortical thickness of the whole-brain and specific brain regions. PreCG, precentral gyrus; SFGdor, superior frontal gyrus (dorsal); ORBsup superior orbital gyrus; MFG, middle frontal gyrus; ORBmid, middle orbital gyrus; IFGoperc, inferior frontal gyrus pars opercularis; IFGtriang, inferior frontal gyrus pars triangularis; ORBinf, inferior orbital gyrus; ROL, rolandic operculum; SMA, supplementary motor area; OLF, olfactory cortex; SFGmed, superior frontal gyrus (medial); ORBsupmed, superior frontal gyrus (medial orbital); REC, gyrus rectus; INS, insula; ACG, anterior cingulate gyrus; DCG, dorsal cingulate gyrus; PCG, posterior cingulate gyrus, $\mathrm{PHG}$, parahippocampal gyrus; CAL, calcarine fissure and surrounding cortex; CUN, cuneus; LING, lingual gyrus; SOG, superior occipital gyrus; MOG, middle occipital gyrus; IOG, inferior occipital gyrus; FFG, fusiform gyrus; PoCG, postcentral gyrus; SPG, superior parietal gyrus; IPL, inferior parietal lobule; SMG, supra marginal gyrus; ANG, angular gyrus; PCUN, precuneus, PCL, paracentral lobule; HES, Heschl's gyrus; STG, superior temporal gyrus; TPOsup, superior temporal pole; MTG, middle temporal gyrus; TPOmid, middle temporal pole; ITG, inferior temporal gyrus.

Additional file 3: Supplementary Figure 2. The correlation between the cortical thickness of each specific brain region and serum NFL in mild cognitive impairment participants $(n=51)$. The correlation between the cortical thickness of each specific brain region and serum NFL is represented in different colors. Positive and negative correlation coefficients are shown in red and blue, respectively. NFL levels were negatively correlated with the cortical thickness of the specific brain regions including the CAL, LING, accumbens, hippocampus, and putamen. PreCG, precentral gyrus; SFGdor, superior frontal gyrus (dorsal); ORBsup superior orbital gyrus; MFG, middle frontal gyrus; ORBmid, middle orbital gyrus; IFGoperc, inferior frontal gyrus pars opercularis; IFGtriang, inferior frontal gyrus pars triangularis; ORBinf, inferior orbital gyrus; ROL, rolandic operculum; SMA, supplementary motor area; OLF, olfactory cortex; SFGmed, superior frontal gyrus (medial); ORBsupmed, superior frontal gyrus (medial orbital); REC, gyrus rectus; INS, insula; ACG, anterior cingulate gyrus; DCG, dorsal cingulate gyrus; PCG, posterior cingulate gyrus, PHG, parahippocampal gyrus; CAL, calcarine fissure and surrounding cortex; CUN, cuneus; LING, lingual gyrus; SOG, superior occipital gyrus; MOG, middle occipital gyrus; IOG, inferior occipital gyrus; FFG, fusiform gyrus; PoCG, postcentral gyrus; SPG, superior parietal gyrus; IPL, inferior parietal lobule; SMG, supra marginal gyrus; ANG, angular gyrus; PCUN, precuneus, PCL, paracentral lobule; HES, Heschl's gyrus; STG, superior temporal gyrus; TPOsup, superior temporal pole; MTG, middle temporal gyrus; TPOmid, middle temporal pole; ITG, inferior temporal gyrus.

Additional file 4: Supplementary Figure 3. The correlation between the cortical thickness of each specific brain region and serum NFL in cognitively unimpaired participants $(n=145)$. The correlation between the cortical thickness of each specific brain region and serum NFL is represented in different colors. Positive and negative correlation coefficients are shown in red and blue, respectively. NFL levels were negatively correlated with the cortical thickness of the whole-brain and specific brain regions including the parietal, temporal, and occipital cortex. PreCG, precentral gyrus; SFGdor, superior frontal gyrus (dorsal); ORBsup superior orbital gyrus; MFG, middle frontal gyrus; ORBmid, middle orbital gyrus; IFGoperc, inferior frontal gyrus pars opercularis; IFGtriang, inferior frontal gyrus pars triangularis; ORBinf, inferior orbital gyrus; ROL, rolandic operculum; SMA, supplementary motor area; OLF, olfactory cortex; SFGmed, superior frontal gyrus (medial); ORBsupmed, superior frontal gyrus (medial orbital); REC, gyrus rectus; INS, insula; ACG, anterior cingulate gyrus; DCG, dorsal cingulate gyrus; PCG, posterior cingulate gyrus, $\mathrm{PHG}$, parahippocampal gyrus; CAL, calcarine fissure and surrounding cortex; CUN, cuneus; LING, lingual gyrus; SOG, superior occipital gyrus; MOG, middle occipital gyrus; IOG, inferior occipital gyrus; FFG, fusiform gyrus; PoCG, postcentral gyrus; SPG, superior parietal gyrus; IPL, inferior parietal lobule; SMG, supra marginal gyrus; ANG, angular gyrus; PCUN, precuneus, $\mathrm{PCL}$, paracentral lobule; HES, Heschl's gyrus; STG, superior temporal gyrus; TPOsup, superior temporal pole; MTG, middle temporal gyrus; TPOmid, middle temporal pole; ITG, inferior temporal gyrus.

Additional file 5: Supplementary Figure 4. Serum and cerebrospinal fluid neurofilament light chain according to amyloid-beta positivity. Serum and cerebrospinal fluid neurofilament light chain according to amyloid-beta positivity. Fit lines are shown for each group. The Spearman's $\rho$ and $P$ values relate to Spearman's rank correlation for each group. NFL, neurofilament light chain; CSF, cerebrospinal fluid.

\section{Authors' contributions}

E-HL and HSK conceptualized and designed study, analyzed the data, interpreted the data, and drafted the manuscript. S-HK and SHC conceptualized and designed study, and revised the manuscript. J-HJ acquired data and analyzed the data. JHJ, J-WJ, KWP, E-JK, HJK, JYH, SJY, and BY acquired data. J-HK, $\mathrm{J}-\mathrm{ML}, \mathrm{H}-\mathrm{HP}$, and $\mathrm{JH}$ reviewed the manuscript. All author(s) read and approved the final manuscript.

\section{Funding}

This research was supported by a grant from the Korea Health Technology R\&D Project through the Korea Health Industry Development Institute, funded by the Ministry of Health \& Welfare, Republic of Korea (grant number: HI20C0253, HU21C0007, HU21C0016, and HU21C0113), and a grant from the Brain Convergence Research Program of the National Research Foundation, funded by the Ministry of Science and ICT, Republic of Korea (NRF-2020M3E5D2A01084721 and NRF-2018M3A9F1023697). 


\section{Availability of data and materials}

The datasets used and analyzed during the current study are available from the corresponding author on reasonable request.

\section{Declarations}

\section{Ethics approval and consent to participate}

The KBASE-V study was approved by obtaining separate IRB review and approval from each institution's ethics committee. Prior to participation in the study, written informed consents were collected from all participants or their legal representatives.

\section{Consent for publication}

Not applicable

\section{Competing interests}

The authors declare that they have no competing interests.

\section{Author details}

${ }^{1}$ Department of Neurology, Hanyang University Guri Hospital, Hanyang University College of Medicine, 153 Gyeongchun-ro, Guri 11923, Republic of Korea. ${ }^{2}$ Department of Neurology, Inha University School of Medicine, 27 Inhang-ro, Jung-gu, Incheon, Republic of Korea. ${ }^{3}$ Department of Neurology, Ewha Womans University School of Medicine, Seoul, Republic of Korea. ${ }^{4}$ Department of Neurology, Kangwon National University School of Medicine, Chuncheon, Republic of Korea. ${ }^{5}$ Department of Neurology, Dong-A Medical Center, Dong-A University College of Medicine, Busan, Republic of Korea. ${ }^{6}$ Department of Neurology, Pusan National University Hospital, Pusan National University School of Medicine and Medical Research Institute, Busan, Republic of Korea. ${ }^{7}$ Department of Neurology, Samsung Medical Center, Sungkyunkwan University School of Medicine, Seoul, Republic of Korea. ${ }^{8}$ Department of Neurology, Yonsei University Wonju College of Medicine, Wonju, Republic of Korea. ${ }^{9}$ Department of Neurology, Eulji University Hospital, Eulji University School of Medicine, Daejeon, Republic of Korea. ${ }^{10}$ Department of Neurology, Konyang University College of Medicine, Daejeon, Republic of Korea. ${ }^{11}$ Department of Pharmacology, Inha University School of Medicine, Incheon, Republic of Korea. ${ }^{12}$ Department of Biomedical Engineering, Hanyang University, Seoul, Republic of Korea. ${ }^{13}$ GemVax \& Kael Co., Ltd., Seongnam, Republic of Korea.

Received: 8 February 2021 Accepted: 14 December 2021 Published online: 07 January 2022

\section{References}

1. Reitz C, Mayeux R. Alzheimer disease: epidemiology, diagnostic criteria, risk factors and biomarkers. Biochem Pharmacol. 2014;88(4):640-51.

2. Sperling RA, Aisen PS, Beckett LA, Bennett DA, Craft S, Fagan AM, et al. Toward defining the preclinical stages of Alzheimer's disease: recommendations from the National Institute on Aging-Alzheimer's Association workgroups on diagnostic guidelines for Alzheimer's disease. Alzheimers Dement. 2011;7(3):280-92.

3. Scheltens P, Blennow K, Breteler MM, de Strooper B, Frisoni GB, Salloway S, et al. Alzheimer's disease. Lancet. 2016;388(10043):505-17.

4. Mattsson N, Andreasson U, Zetterberg H, Blennow K. Association of plasma neurofilament light with neurodegeneration in patients with Alzheimer disease. JAMA Neurol. 2017;74(5):557-66.

5. Skillback T, Zetterberg H, Blennow K, Mattsson N. Cerebrospinal fluid biomarkers for Alzheimer disease and subcortical axonal damage in 5,542 clinical samples. Alzheimers Res Ther. 2013;5(5):47.

6. Preische O, Schultz SA, Apel A, Kuhle J, Kaeser SA, Barro C, et al. Serum neurofilament dynamics predicts neurodegeneration and clinical progression in presymptomatic Alzheimer's disease. Nat Med. 2019;25(2):277-83.

7. Forgrave LM, Ma M, Best JR, DeMarco ML. The diagnostic performance of neurofilament light chain in CSF and blood for Alzheimer's disease, frontotemporal dementia, and amyotrophic lateral sclerosis: A systematic review and meta-analysis. Alzheimers Dement. 2019;11:730-43.

8. Hwang J, Jeong JH, Yoon SJ, Park KW, Kim EJ, Yoon B, et al. Clinical and biomarker characteristics according to clinical spectrum of Alzheimer's disease (AD) in the validation cohort of Korean brain aging study for the early diagnosis and prediction of AD. J Clin Med. 2019;8(3):341.

9. Lee DY, Lee KU, Lee JH, Kim KW, Jhoo JH, Kim SY, et al. A normative study of the CERAD neuropsychological assessment battery in the Korean elderly. J Int Neuropsychol Soc. 2004;10(1):72-81.

10. Choi SH, Lee BH, Kim S, Hahm DS, Jeong JH, Yoon SJ, et al. Interchanging scores between clinical dementia rating scale and global deterioration scale. Alzheimer Dis Assoc Disord. 2003;17(2):98-105.

11. Jang JW, Kim Y, Choi YH, Lee JM, Yoon B, Park KW, et al. Association of nutritional status with cognitive stage in the elderly Korean population: The Korean brain aging study for the early diagnosis and prediction of Alzheimer's disease. J Clin Neurol. 2019;15(3):292-300.

12. Albert MS, DeKosky ST, Dickson D, Dubois B, Feldman HH, Fox NC, et al. The diagnosis of mild cognitive impairment due to Alzheimer's disease: recommendations from the National Institute on Aging-Alzheimer's Association workgroups on diagnostic guidelines for Alzheimer's disease. Alzheimers Dement. 2011;7(3):270-9.

13. Petersen RC, Doody R, Kurz A, Mohs RC, Morris JC, Rabins PV, et al. Current concepts in mild cognitive impairment. Arch Neurol. 2001;58(12):1985-92.

14. Chin J, Park J, Yang SJ, Yeom J, Ahn Y, Baek MJ, et al. Re-standardization of the Korean-Instrumental Activities of Daily Living (K-IADL): clinical usefulness for various neurodegenerative diseases. Demen Neurocognitive Disord. 2018;17(1):11-22.

15. Bae JN, Cho MJ. Development of the Korean version of the Geriatric Depression Scale and its short form among elderly psychiatric patients. J Psychosom Res. 2004;57(3):297-305.

16. Blessed G, Tomlinson BE, Roth M. The association between quantitative measures of dementia and of senile change in the cerebral grey matter of elderly subjects. Br J Psychiatry. 1968;114(512):797-811.

17. Koh SH, Choi SH, Jeong JH, Jang JW, Park KW, Kim EJ, et al. Telomere shortening reflecting physical aging is associated with cognitive decline and dementia conversion in mild cognitive impairment due to Alzheimer's disease. Aging. 2020;12(5):4407-23.

18. Beckett LA, Donohue MC, Wang C, Aisen P, Harvey DJ, Saito N. The Alzheimer's disease neuroimaging initiative phase 2: increasing the length, breadth, and depth of our understanding. Alzheimers Dement. 2015;11(7):823-31.

19. Zijdenbos AP, Forghani R, Evans AC. Automatic "pipeline" analysis of 3-D MRI data for clinical trials: application to multiple sclerosis. IEEE Trans Med Imaging. 2002;21(10):1280-91.

20. Sled JG, Zijdenbos AP, Evans AC. A nonparametric method for automatic correction of intensity nonuniformity in MRI data. IEEE Trans Med Imaging. 1998;17(1):87-97.

21. Collins DL, Neelin P, Peters TM, Evans AC. Automatic 3D intersubject registration of MR volumetric data in standardized Talairach space. J Comput Assist Tomogr. 1994;18(2):192-205.

22. Smith SM. Fast robust automated brain extraction. Hum Brain Mapp. 2002;17(3):143-55.

23. Kim JS, Singh V, Lee JK, Lerch J, Ad-Dab'bagh Y, MacDonald D, et al. Automated 3-D extraction and evaluation of the inner and outer cortical surfaces using a Laplacian map and partial volume effect classification. Neurolmage. 2005;27(1):210-21.

24. Kabani N, Le Goualher G, MacDonald D, Evans AC. Measurement of cortical thickness using an automated 3-D algorithm: a validation study. Neurolmage. 2001;13(2):375-80.

25. Patenaude B, Smith SM, Kennedy DN, Jenkinson M. A Bayesian model of shape and appearance for subcortical brain segmentation. Neurolmage. 2011;56(3):907-22.

26. Jack CR Jr, Bennett DA, Blennow K, Carrillo MC, Dunn B, Haeberlein SB, et al. NIA-AA Research Framework: toward a biological definition of Alzheimer's disease. Alzheimers Dement. 2018;14(4):535-62.

27. Klunk WE, Koeppe RA, Price JC, Benzinger TL, Devous MD Sr, Jagust WJ, et al. The Centiloid project: standardizing quantitative amyloid plaque estimation by PET. Alzheimers Dement. 2015;11(1):1-15.e11-14.

28. de Souza GS, Andrade MA, Borelli WV, Schilling LP, Matushita CS, Portuguez MW, et al. Amyloid- $\beta$ PET classification on cognitive aging stages using the Centiloid scale. Mol Imaging Biol. 2021. https://doi.org/10.1007/ s11307-021-01660-7. Epub 2021 Oct 5.

29. Amadoru S, Doré V, McLean CA, Hinton F, Shepherd CE, Halliday GM, et al. Comparison of amyloid PET measured in Centiloid units with 
neuropathological findings in Alzheimer's disease. Alzheimers Res Ther. 2020;12(1):22.

30. Association AP. Diagnostic and statistical manual of mental disorders: DSM-IV-TR. Washington DC. USA. 2000.

31. McKhann GM, Knopman DS, Chertkow H, Hyman BT, Jack CR Jr, Kawas $\mathrm{CH}$, et al. The diagnosis of dementia due to Alzheimer's disease: recommendations from the National Institute on Aging-Alzheimer's Association workgroups on diagnostic guidelines for Alzheimer's disease. Alzheimers Dement. 2011;7(3):263-9.

32. Lopez OL, Schwam E, Cummings J, Gauthier S, Jones R, Wilkinson D, et al. Predicting cognitive decline in Alzheimer's disease: an integrated analysis. Alzheimers Dement. 2010;6(6):431-9.

33. Chaves ML, Camozzato AL, Köhler C, Kaye J. Predictors of the progression of dementia severity in Brazilian patients with Alzheimer's disease and vascular dementia. Int J Alzheimers Dis. 2010;2010:673581.

34. Csernansky JG, Wang L, Swank J, Miller JP, Gado M, McKeel D, et al. Preclinical detection of Alzheimer's disease: hippocampal shape and volume predict dementia onset in the elderly. Neurolmage. 2005;25(3):783-92.

35. Solfrizzi V, Panza F, Colacicco AM, D'Introno A, Capurso C, Torres F, et al. Vascular risk factors, incidence of $\mathrm{MCl}$, and rates of progression to dementia. Neurology. 2004;63(10):1882-91.

36. Mattsson N, Cullen NC, Andreasson U, Zetterberg H, Blennow K. Association between longitudinal plasma neurofilament light and neurodegeneration in patients with Alzheimer disease. JAMA Neurol. 2019;76(7):791-9.

37. Fish PV, Steadman D, Bayle ED, Whiting P. New approaches for the treatment of Alzheimer's disease. Bioorg Med Chem Lett. 2019;29(2):125-33.

38. Brureau A, Blanchard-Bregeon V, Pech C, Hamon S, Chaillou P, Guillemot $J C$, et al. NF-L in cerebrospinal fluid and serum is a biomarker of neuronal damage in an inducible mouse model of neurodegeneration. Neurobiol Dis. 2017:104:73-84.

39. Lee MK, Cleveland DW. Neuronal intermediate filaments. Annu Rev Neurosci. 1996;19:187-217.

40. Petzold A. Neurofilament phosphoforms: surrogate markers for axonal injury, degeneration and loss. J Neurol Sci. 2005;233(1-2):183-98.

41. Mielke MM, Syrjanen JA, Blennow K, Zetterberg H, Vemuri P, Skoog I, et al. Plasma and CSF neurofilament light: Relation to longitudinal neuroimaging and cognitive measures. Neurology. 2019;93(3):e252-60.

42. Meeter LH, Dopper EG, Jiskoot LC, Sanchez-Valle R, Graff C, Benussi L, et al. Neurofilament light chain: a biomarker for genetic frontotemporal dementia. Ann Clin TransI Neurol. 2016;3(8):623-36.

43. Alzheimer's disease neuroimaging initiative 3 (ADNI3) protocol. 2016 https://clinicaltrials.gov/ct2/show/NCT02854033. Accessed 11 Nov 2021.

\section{Publisher's Note}

Springer Nature remains neutral with regard to jurisdictional claims in published maps and institutional affiliations.

Ready to submit your research? Choose BMC and benefit from:

- fast, convenient online submission

- thorough peer review by experienced researchers in your field

- rapid publication on acceptance

- support for research data, including large and complex data types

- gold Open Access which fosters wider collaboration and increased citations

- maximum visibility for your research: over $100 \mathrm{M}$ website views per year

At BMC, research is always in progress.

Learn more biomedcentral.com/submissions 